\title{
Maximize Expected Profits by Dynamic After-Sales Service Investment Strategy Based on Word-of-Mouth Marketing in Social Network Shopping
}

\author{
Ying Yu $\mathbb{D}^{1,2}$ Jiaomin Liu, ${ }^{1}$ Jiadong Ren $\mathbb{D}^{1,3}$ Qian Wang, ${ }^{1,3}$ and Cuiyi Xiao ${ }^{4}$ \\ ${ }^{1}$ College of Information Science and Engineering, Yanshan University, Qinhuangdao, Hebei, China \\ ${ }^{2}$ Liren College of Yanshan University, Qinhuangdao, Hebei, China \\ ${ }^{3}$ Computer Virtual Technology and System Integration Laboratory of Hebei Province, Qinhuangdao, China \\ ${ }^{4}$ College of Mathematics and Information Technology, Hebei Normal University of Science and Technology, \\ Qinhuangdao, Hebei, China \\ Correspondence should be addressed to Jiadong Ren; jdren@ysu.edu.cn
}

Received 4 August 2021; Accepted 1 October 2021; Published 11 November 2021

Academic Editor: Sameh S. Askar

Copyright ( 92021 Ying Yu et al. This is an open access article distributed under the Creative Commons Attribution License, which permits unrestricted use, distribution, and reproduction in any medium, provided the original work is properly cited.

\begin{abstract}
This paper discusses how word-of-mouth marketing affects the profits of product sales in social network-based shopping under good after-sales service. First, a new word-of-mouth communication model based on silent evaluation, positive evaluation, and negative evaluation is proposed. Second, we use the way of increasing after-sales service to achieve high praise and thereby maximize the expected profits. Thus, the proportion control problem of after-sales service investment is modeled as an optimal control problem. Third, the existence of optimal control is proved, and an optimal control strategy for dynamic proportion of after-sales service investment is proposed. Fourth, through data simulation of different real-world networks, it is verified that the expected profits under the dynamic after-sales service strategy is higher than that under any uniform control strategy. Finally, sensitivity analysis is performed to explore how different parameters affect the expected profits.
\end{abstract}

\section{Introduction}

With the boom of social networking, more and more consumers are making purchase decisions based on word of mouth (WOM). Nearly three-quarters (73\%) of consumers regularly recommend products to their friends, according to an Accenture survey [1]. The rapid advancement of e-commerce and logistics networks has changed the way of shopping in China. Online shopping is characterized by easy access to actual WOM. Compared with traditional advertising strategies, WOM influence strategies cost less and deliver more revenue [2-9].

In recent years, researchers have examined ways to maximize product profits from different perspectives based on WOM marketing. For example, the relationship between users was used to explore the impact of WOM marketing on consumers [10-12]. Users' WOM feedback data was used to mine information that is conducive to enterprises' innovation or growth [13-15]. A dynamic discount pricing strategy aiming at maximizing product profits based on WOM marketing was proposed [16-18]. Using WOM as an epidemic, a framework was constructed to discuss the impact of WOM on sales based on epidemic models [19-21].

In recent years, social network-based shopping, typified by shopping on WeChat, has emerged in China. The biggest characteristic of this shopping lies in its reliance on social networks for product sales. In particular, since the early 2020, when the COVID-19 outbreak caused a sharp drop in customer visits and product sales in physical stores, more and more sellers have begun to set up shopping groups to sell products on social networks such as WeChat. These shopping groups, which we call community buying groups, tend to have the following characteristics: 
Members in such a group are often closely related, i.e., they may be members of the same company, neighbor users, etc. This makes it easy to spread WOM among different nodes within a social network, which directly affects product sales. Since most customers are familiar with each other, reviews are usually truthful and valid. Therefore, positive WOM has a significant impact on product sales.

Community group buying often involves repeat purchases, and positive WOM, in particular, prompts consumers to make repeat purchases. Therefore, WOM affects the initial purchase intention of customers.

After customers buy a product, their evaluation is usually three-fold: silent, positive, and negative. Evidently, positive WOM helps to increase the purchase intention of the remaining customers, and negative WOM reduces their purchase intention.

After-sales service works in two ways for enhancing WOM. On the one hand, it encourages customers who have been remaining silent to post more positive reviews; on the other hand, it helps to reverse customers' negative reviews and finally drive positive WOM publicity.

We note that several studies have been conducted on the positive and negative effects of WOM [22-26]. Based on the cognitive dissonance theory and social support theory, Balaji et al. [27] studied the roles of situational factors, personal factors, and social network factors in determining customers' willingness to use social networking sites for negative WOM communication. Verhagen et al. [28] proposed a sender-oriented model to explore the effects of emotions and negative online WOM on re-sponsorship and switching intention. Dalman et al. [29] investigated how high-equity and low-equity brands attract negative WOM from consumers in the event of market failure. Weitzl et al. [30] suggested that online care (i.e., messages in response to online complaints) can mitigate complainants' adverse attributions of failure (i.e., track, controllability, and stability).

Inspired by the epidemic model, this paper takes WOM as a kind of epidemic and establishes a nodal dynamic WOM propagation model. From the characteristics of community group buying, it can be concluded that WOM has important implications for product sales. However, community group buying is small in scale and the profit it generates is limited. Investing heavy resources to maintain WOM would be costly. In this paper, we focus on how to dynamically invest in after-sales service to maximize profits. The main contributions are as follows:

First, a node-level model based on WOM marketing is established to reveal the influence of WOM on consumers' purchase intention.

Second, on this basis, the dynamic control problem of WOM through after-sales service is modeled as an optimal control problem.
Third, we prove that the model has optimal control and obtain the optimal system for solving the model.

Fourth, by solving the corresponding optimal system, some optimal control strategies are given, which are then compared with uniform control. The comparison demonstrates the superiority of optimal control strategies over uniform control ones.

Finally, the influence of after-sales service on product sales is further discussed through sensitivity analysis of relevant after-sales service parameters.

The remainder of the paper is organized as follows. In Section 2, we establish a node-level Target-Buying-Refusing (TBR) model based on WOM marketing and model the dynamic after-sales service (DAS) problem as an optimal control problem. In Section 3, we perform theoretical analysis on the optimal control problem and give a dynamic strategy for after-sales service investment. Some optimal DAS strategies are given in Section 4. In Section 5, the influence of some after-sales service parameters on expected profits is further revealed. The concluding remarks are drawn in Section 6.

\section{The Modeling of the DAS Investment Problem}

In this section, we consider the WOM marketing problem with DAS investment. In view of the sales activities involved in community group buying, a DAS investment strategy is developed in order to maximize profits for sellers. The highlights of this section are as follows: (1) introducing basic symbols and terms; (2) establishing a TBR model based on WOM marketing; (3) modeling the DAS problem as an optimal control problem.

2.1. Terms and Notations. Assuming the number of users for a community group purchase is $N$, we let $G=(V, E)$ denote the topology of this network. Specifically, $V=\left(V_{1}, V_{2}, \ldots, V_{N}\right)$ represents the nodes of the network and $N$ is the number of nodes in the network; furthermore, $E_{i j}=\left\{V_{i}, V_{j}\right\} \in E$, which represents nodes $V_{i}$ and $V_{j}$ are friends, so $G$ is an undirected network; $A=\left(a_{i j}\right)_{N \times N}$ represents the adjacency matrix of network $G$. When $a_{i j}=1, V_{i}$ and $V_{j}$ can share their reviews on products with each other.

Let the vector $O(t)=\left(O_{1}(t), O_{2}(t), \ldots, O_{N}(t)\right)$ represent the state of $N$ nodes in the network at time $t$. Assuming that a product is sold in a finite time horizon $[0, T]$, individual users may have the following three states in community group buying: $O_{i}(t)=0, O_{i}(t)=1$, and $O_{i}(t)=2$, corresponding to target customers (who have not yet decided whether to buy the product), buying customers (who buy the product), and refusing customers (who refuse to buy the product) at time $t$, respectively. Let $T_{i}(t), B_{i}(t)$, and $R_{i}(t)$ denote the expected probabilities of node $V_{i}$ being target customers, buying customers, and refusing customers at time $t$, respectively: 


$$
\begin{aligned}
& T_{i}(t)=\operatorname{Pr}\left\{O_{i}(t)=0\right\}, \\
& B_{i}(t)=\operatorname{Pr}\left\{O_{i}(t)=1\right\}, \\
& R_{i}(t)=\operatorname{Pr}\left\{O_{i}(t)=2\right\} .
\end{aligned}
$$

Note that $T_{i}(t)+B_{i}(t)+R_{i}(t)=1$. Specifically, buying customers are categorized into three groups in terms of their evaluation: silent, positive, and negative. Let $S_{i}(t), P_{i}(t)$, and $N_{i}(t)$ represent the expected probabilities of buying customers who remain silent and give positive and negative evaluations at time $t$, respectively.

To solve the WOM propagation problem with DAS investment, we introduce the following assumptions:

Due to the shopping share of neighbor nodes who buy the products, target customers become buying customers with a probability of $\rho$ on average

Due to the shopping share of neighbor nodes who refuse to buy the products, target customers become refusing customers with a probability of $\gamma$ on average

For buying customers, $\alpha_{1}, \alpha_{2}$, and $\alpha_{3}$ represent the probabilities of them remaining silent, giving positive evaluation and giving negative evaluation; hence, $\alpha_{1}+\alpha_{2}+\alpha_{3}=1$

Due to the positive WOM effect of buyers, target customers become buying customers with a probability of $\beta_{1}$ on average

Due to the negative WOM effect of buyers, target customers become refusing customers with a probability of $\beta_{2}$ on average

The above assumptions are independent of each other, and the state transition diagram of the node is shown in Figure 1. In practice, parameters $\rho, \gamma, \alpha_{1}, \alpha_{2}, \alpha_{3}, \beta_{1}, \beta_{2}$ can be estimated from the information of the network.

The vector,

$$
\mathbf{E}(t)=(T(t), B(t), R(t))
$$

represents the expected state of nodes in the network at time $t$, where

$$
\left\{\begin{array}{l}
T(t)=\left(T_{1}(t), T_{2}(t), \ldots, T_{N}(t)\right), \\
B(t)=\left(B_{1}(t), B_{2}(t), \ldots, B_{N}(t)\right), \\
R(t)=\left(R_{1}(t), R_{2}(t), \ldots, R_{N}(t)\right) .
\end{array}\right.
$$

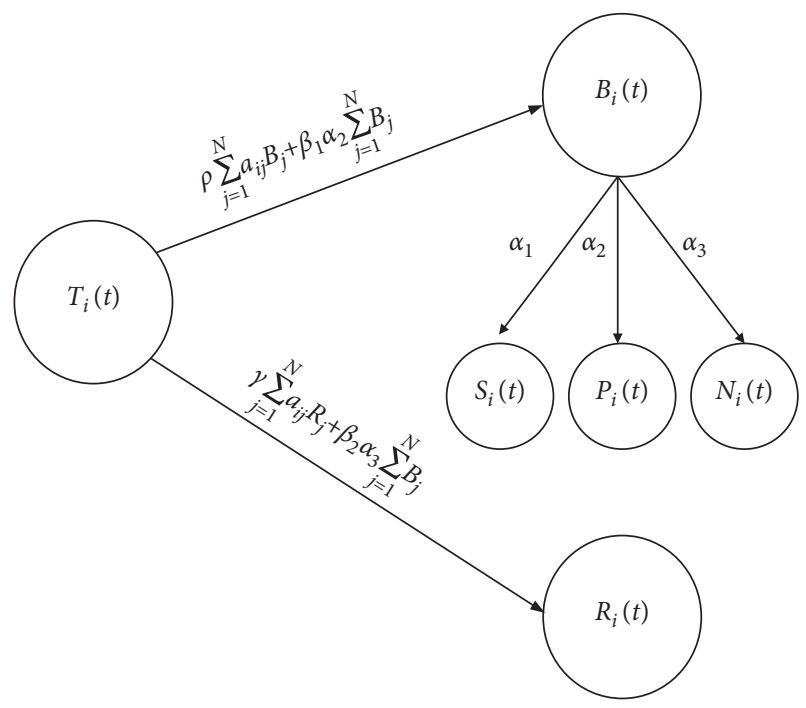

FIgURE 1: State transition diagram of individual $V_{i}$ at time $t$.

For ease of understanding, a simple example is given. Assume that there is a topological relationship between network nodes, as shown in Figure 2, and the probability that node 1 is a target customer is 1 at time $t$, i.e., $T_{1}(t)=\operatorname{Pr}\left(O_{1}(t)=0\right)=1$. Suppose the adjacency matrix of nodes in the network is

$$
A=\left(\begin{array}{llllll}
0 & 1 & 1 & 1 & 0 & 0 \\
1 & 0 & 1 & 1 & 1 & 0 \\
1 & 1 & 0 & 0 & 0 & 1 \\
1 & 1 & 0 & 0 & 0 & 0 \\
0 & 1 & 0 & 0 & 0 & 0 \\
0 & 0 & 1 & 0 & 0 & 0
\end{array}\right) .
$$

The probabilities of network nodes being in each state are shown in Table 1.

Parameter values are shown in Table 2.

Then, the probability that node 1 is a buying node at $t+\Delta t$ is

$$
\begin{aligned}
B_{1}(t+\Delta t) & =\operatorname{Pr}\left(O_{1}(t+\Delta t)=1\right)=\rho \sum_{j=1}^{N} a_{i j} B_{j}+\beta_{1} \alpha_{2} \sum_{j=1}^{N} B_{j} \\
& =\rho\left(a_{12} \cdot B_{2}+a_{13} \cdot B_{3}+a_{14} \cdot B_{4}\right)+\beta_{1} \alpha_{2}\left(B_{1}+B_{2}+B_{3}+B_{4}+B_{5}+B_{6}\right) \\
& =0.1 \cdot(0.7+0.7+0.2)+0.02 \cdot(0.7+0.7+0.2+0.6+0.2)=0.208 .
\end{aligned}
$$




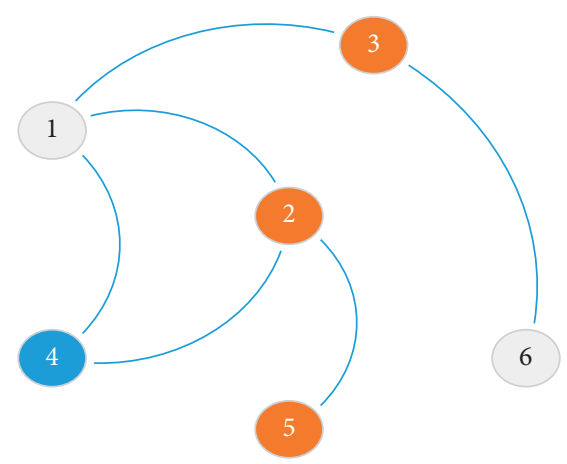

Figure 2: A simple network topology.

TABle 1: Probabilities of nodes being in each state.

\begin{tabular}{lccc}
\hline Node number & Probability of $T_{i}$ & Probability of $B_{i}$ & Probability of $R_{i}$ \\
\hline 2 & 0.2 & 0.7 & 0.1 \\
3 & 0.1 & 0.7 & 0.2 \\
4 & 0.2 & 0.2 & 0.6 \\
5 & 0.3 & 0.6 & 0.1 \\
6 & 0.7 & 0.2 & 0.1 \\
\hline
\end{tabular}

Then, the probability that node 1 is a refusing node at $t+$ $\Delta t$ is

$$
\begin{aligned}
R_{1}(t+\Delta t) & =\operatorname{Pr}\left(O_{1}(t+\Delta t)=2\right)=\gamma \sum_{j=1}^{N} a_{i j} R_{j}+\beta_{2} \alpha_{3} \sum_{j=1}^{N} B_{j} \\
& =\gamma\left(a_{12} \cdot R_{2}+a_{13} \cdot R_{3}+a_{14} \cdot R_{4}\right)+\beta_{2} \alpha_{3}\left(B_{1}+B_{2}+B_{3}+B_{4}+B_{5}+B_{6}\right) \\
& =0.1 \cdot(0.1+0.2+0.6)+0.04 \cdot(0.7+0.7+0.2+0.6+0.2)=0.186 .
\end{aligned}
$$
is

Then, the probability that node 1 is a target node at $t+\Delta t$

$$
T_{1}(t+\Delta t)=1-0.208-0.186=0.606
$$

2.2. Dynamic TBR Model Based on WOM Marketing. The TBR model based on WOM marketing to be derived later conforms to the above assumption. The following system gives an equivalent form of the exact TBR model:

$$
\left\{\begin{array}{l}
\frac{\mathrm{d} T_{i}(t)}{\mathrm{d} t}=-\left[\rho \sum_{j=1}^{N} a_{i j} B_{j}(t)+\beta_{1} \alpha_{2} \sum_{j=1}^{N} B_{j}(t)+\gamma \sum_{j=1}^{N} a_{i j} R_{j}(t)+\beta_{2} \alpha_{3} \sum_{j=1}^{N} B_{j}(t)\right] T_{i}(t), \\
\frac{\mathrm{d} B_{i}(t)}{\mathrm{d} t}=\left[\rho \sum_{j=1}^{N} a_{i j} B_{j}(t)+\beta_{1} \alpha_{2} \sum_{j=1}^{N} B_{j}(t)\right] T_{i}(t), \\
\frac{\mathrm{d} R_{i}(t)}{\mathrm{d} t}=\left[\gamma \sum_{j=1}^{N} a_{i j} R_{j}(t)+\beta_{2} \alpha_{3} \sum_{j=1}^{N} B_{j}(t)\right] T_{i}(t),
\end{array}\right.
$$


TABLE 2: Values of parameters based on network.

\begin{tabular}{lccccccc}
\hline Parameter & $\rho$ & $\gamma$ & $\alpha_{1}$ & $\alpha_{2}$ & $\alpha_{3}$ & $\beta_{1}$ & $\beta_{2}$ \\
\hline Value & 0.1 & 0.2 & 0.4 & 0.2 & 0.4 & 0.1 & 0.1 \\
\hline
\end{tabular}

under the initial condition $\mathbf{E}(0)=\mathbf{E}_{0}$.

System (8) may be written in system as

$$
\left\{\begin{array}{l}
\frac{\mathrm{d} \mathbf{E}(t)}{\mathrm{d} t}=\mathbf{f}_{1}(\mathbf{E}(t)), \quad 0 \leq t \leq T, \\
\mathbf{E}(0)=\mathbf{E}_{0} .
\end{array}\right.
$$

2.3. Optimal Control Modeling of the DAS Problem. We refer to the function $\theta=\left\{\theta_{1}(t), \theta_{2}(t)\right\}, 0 \leq t \leq T$, as a DAS strategy. $\theta_{1}(t)$ is the proportion of investment in the first type of after-sales service, including the proportion of silent users converted into positive reviews through cash back. $\theta_{2}(t)$ is the proportion of investment in the second type of after-sales service, including the proportion of negative reviews converted into positive ones by compensating users for their losses. Therefore, we assume that the DAS strategy is

$$
\theta=\left\{\left(\theta_{1}(t), \theta_{2}(t)\right) \in L[0, T]^{2} \mid 0 \leq \theta_{1}(t) \leq \bar{\theta}_{1}, \quad 0 \leq \theta_{2} \leq \bar{\theta}_{2}, 0 \leq t \leq T\right\},
$$

where $L[0, T]^{2}$ represents the set of all Lebesgue integrable functions defined on $[0, T][31]$.

It is widely known that profits are closely associated with the sales of products. Theoretically, the higher the sales of products is, the more negative reviews there will be. In WOM-oriented community group buying, in particular, negative reviews will directly affect the sales of products, resulting in a decline in profits. The proportion of positive WOM can be increased by investing in after-sales service. However, such investment tends to increase the cost and reduce the profit. In view of this, this paper intends to establish a dynamic proportion of investment in after-sales service to maximize the final profit.

Remark 1. Assuming that the profit per unit of a product is $c_{1}$, the total profit from selling the product at time $[0, T]$ is

$$
L_{1}(\theta)=\int_{0}^{T} \sum_{i=1}^{N} c_{1} B_{i}(t) \mathrm{d} t
$$

Remark 2. Assuming that the investment proportion of the first type of after-sales service is $\theta_{1}(t)$ and its average unit cost is $c_{2}$, the cost of after-sales service at time $[0, T]$ is

$$
L_{2}(\theta)=\int_{0}^{T} \sum_{i=1}^{N} c_{2} \alpha_{1} \theta_{1}(t) B_{i}(t) \mathrm{d} t
$$

Remark 3. Assuming that the investment proportion of the second type of after-sales service is $\theta_{2}(t)$ and its average unit cost is $c_{3}$, the cost of after-sales service at time $[0, T]$ is

$$
L_{3}(\theta)=\int_{0}^{T} \sum_{i=1}^{N} c_{3} \alpha_{3} \theta_{2}(t) B_{i}(t) \mathrm{d} t .
$$

In summary, the expected profit is

$$
\begin{aligned}
J(\theta) & =L_{1}(\theta)-L_{2}(\theta)-L_{3}(\theta) \\
& =\int_{0}^{T} \sum_{i=1}^{N}\left(c_{1}-c_{2} \alpha_{1} \theta_{1}(t)-c_{3} \alpha_{3} \theta_{2}(t)\right) B_{i}(t) \mathrm{d} t \\
& \equiv \int_{0}^{T} \mathbf{F}(\mathbf{E}(t), \theta(t)) \mathrm{d} t
\end{aligned}
$$

where

$$
\mathbf{F}(\mathbf{E}(t), \theta(t))=\sum_{i=1}^{N}\left(c_{1}-c_{2} \alpha_{1} \theta_{1}(t)-c_{3} \alpha_{3} \theta_{2}(t)\right) B_{i}(t)
$$

Based on the above discussions and assumptions, this DAS problem can be modeled as an optimal control problem as follows: 


$$
\max _{\theta \in \Theta}=\int_{0}^{T} \mathbf{F}(\mathbf{E}(t), \theta(t)) \mathrm{d} t
$$

\section{subject to}

$$
\left\{\begin{array}{l}
\frac{\mathrm{d} T_{i}(t)}{\mathrm{d} t}=\left[\rho \sum_{j=1}^{N} a_{i j} B_{j}(t)+\beta_{1}\left(\alpha_{2}+\alpha_{1} \theta_{1}+\alpha_{3} \theta_{2}\right) \sum_{j=1}^{N} B_{j}(t)\right. \\
\left.+\gamma \sum_{j=1}^{N} a_{i j} R_{j}(t)+\beta_{2}\left(\alpha_{3}\left(1-\theta_{2}\right)\right) \sum_{j=1}^{N} B_{j}(t)\right] T_{i}(t), \\
\frac{\mathrm{d} B_{i}(t)}{\mathrm{d} t}=\left[\rho \sum_{j=1}^{N} a_{i j} B_{j}(t)+\beta_{1}\left(\alpha_{2}+\alpha_{1} \theta_{1}+\alpha_{3} \theta_{2}\right) \sum_{j=1}^{N} B_{j}(t)\right] T_{i}(t), \\
\frac{\mathrm{d} R_{i}(t)}{\mathrm{d} t}=\left[\gamma \sum_{j=1}^{N} a_{i j} R_{j}(t)+\beta_{2}\left(\alpha_{3}\left(1-\theta_{2}\right)\right) \sum_{j=1}^{N} B_{j}(t)\right] T_{i}(t), \\
\mathbf{E}(0)=\mathbf{E}_{0} .
\end{array}\right.
$$

The associated TBR model based on WOM can be rewritten as

$$
\left\{\begin{array}{l}
\frac{\mathrm{d} \mathbf{E}(t)}{\mathrm{d} t}=\mathbf{f}_{2}(\mathbf{E}(t), \theta(t)), \quad 0 \leq t \leq T, \\
\mathbf{E}(0)=\mathbf{E}_{0}
\end{array}\right.
$$

We refer to this optimal control problem as the DAS problem. This model can be described as a 15-tuple problem as follows:

$$
\Phi=\left(G, \rho, \gamma, \alpha_{1}, \alpha_{2}, \alpha_{3}, \beta_{1}, \beta_{2}, \bar{\theta}_{1}, \bar{\theta}_{2}, c_{1}, c_{2}, c_{3}, \mathbf{E}_{0}, T\right) .
$$

\section{Theoretical Study of the DAS Control Problem}

3.1. Solvability of the DAS Problem. First, we prove that the DAS problem is an optimal control problem and is solvable. Hence, we derive Lemma 1 [32].

Lemma 1. The DAS problem is an optimal control problem if all the following five conditions hold.

(1) $\Theta$ is convex and closed

(2) There exists $\theta \in \Theta$ such that system (17) is solvable
(3) $\mathbf{f}_{2}(\mathbf{E}, \theta)$ is bounded by a linear function in $\mathbf{E}$

(4) $\mathbf{F}(\mathbf{E}, \theta)$ is convex on $\Theta$

(5) There exists $\delta>1, d_{1}>0$, and $d_{2}$ such that $\mathbf{F}(\mathbf{E}, \theta) \geq\|\theta\|_{2}^{\delta}+d_{2}$

Then, we can deduce the following theorem.

Theorem 1. The DAS problem is an optimal control problem.

Proof. First, let $\theta=\left(\theta_{1}(t), \theta_{2}(t)\right)$ be a limit point of $\Theta$. Then, there exists a sequence $\theta^{n}(t)=\left(\theta_{1}^{n}(t), \theta_{2}^{n}(t)\right), n=1,2, \ldots$, of points of $\Theta$, which approaches $\theta(t)$. Since

$$
\begin{aligned}
& 0 \leq \theta_{1}(t)=\lim _{n \longrightarrow \infty} \theta_{1}^{(n)}(t) \leq \bar{\theta}_{1} \leq 1, \\
& 0 \leq \theta_{2}(t)=\lim _{n \longrightarrow \infty} \theta_{2}^{(n)}(t) \leq \bar{\theta}_{2} \leq 1, \quad 0 \leq t \leq T .
\end{aligned}
$$

$\Theta$ is closed. Let $\theta^{(1)}, \theta^{(2)} \in \Theta, \quad 0<\eta<1, \widehat{\theta}=$ $(1-\eta) \theta^{(1)}+\eta \theta^{(2)}$. As $L[0, T]^{2}$ is a real vector space, we have $\widehat{\theta} \in L[0, T]^{2} \quad$ and $0 \leq(1-\eta) \theta^{(1)}(t)+\eta \theta^{(2)} \leq(1-\eta)$ $\bar{\theta}+\eta \bar{\theta}=\bar{\theta}$. Hence, $\Theta$ is convex. Second, let $\theta^{*}(t) \in \Theta$. As $\mathbf{f}_{2}\left(\mathbf{E}, \theta^{*}\right)$ is continuously differentiable, it follows by the continuation theorem for differentiable systems [33] that the corresponding state evolution state is solvable.

Third, it follows from (17) that for $1 \leq i \leq N$. As $T_{i}(t)=$ $1-B_{i}(t)-R_{i}(t)$ and $0 \leq \theta(t) \leq 1$, then we have 


$$
\begin{aligned}
&-\left(\rho \sum_{j=1}^{N} a_{i j} B_{j}(t)+\beta_{1} \sum_{j=1}^{N} B_{j}(t)+\gamma \sum_{j=1}^{N} a_{i j} R_{j}(t)+\beta_{2} \alpha_{3} \sum_{j=1}^{N} B_{j}(t)\right) \leq \frac{\mathrm{d} T_{i}}{\mathrm{~d} t} \leq \beta_{2} \alpha_{3} \sum_{j=1}^{N} B_{j}, \\
&\left(\rho \sum_{j=1}^{N} a_{i j} B_{j}(t)+\beta_{1} \alpha_{2} \sum_{j=1}^{N} B_{j}(t)\right) T_{i} \leq \frac{\mathrm{d} B_{i}}{\mathrm{~d} t} \leq\left(\rho \sum_{j=1}^{N} a_{i j} B_{j}(t)+\beta_{1} \sum_{j=1}^{N} B_{j}(t)\right) T_{i}, \\
&\left(\gamma \sum_{j=1}^{N} a_{i j} R_{j}(t)\right) T_{i} \leq \frac{\mathrm{d} R_{i}}{\mathrm{~d} t} \leq\left(\gamma \sum_{j=1}^{N} a_{i j} R_{j}(t)+\beta_{2} \alpha_{3} \sum_{j=1}^{N} B_{j}(t)\right) T_{i} .
\end{aligned}
$$

Hence, $\mathbf{f}_{2}(\mathbf{E}, \theta)$ is bounded by a linear function in $\mathbf{E}$. The fourth condition follows that $\mathbf{F}(\mathbf{E}, \theta)$ is linear in $\Theta$ and is therefore convex.

Finally, $\mathbf{F}(\mathbf{E}, \theta) \geq 0 \geq\left(\theta_{1}^{2}+\theta_{2}^{2}\right)-\left(\bar{\theta}_{1}^{2}+\bar{\theta}_{2}^{2}\right)=\|\theta\|_{2}^{2}-\left(\bar{\theta}_{1}^{2}+\right.$ $\left.\bar{\theta}_{2}^{2}\right)$. Then, there exists $d_{1}=1, \delta=2, d_{2}=-\left(\bar{\theta}_{1}^{2}+\bar{\theta}_{2}^{2}\right)$ such that $\mathbf{F}(\mathbf{E}, \theta) \geq d_{1}\|\theta\|_{2}^{\delta}+d_{2}$. By Lemma 1 , Theorem 1 is proved.

3.2. The Optimality System. According to the optimal control theory, the Hamiltonian function of the DAS problem is

$$
\begin{aligned}
H(\mathbf{E}(t), \theta(t), X(t))= & \sum_{i=1}^{N}\left(c_{1}-c_{2} \alpha_{1} \theta_{1}-c_{3} \alpha_{3} \theta_{2}\right) B_{i}(t) \\
& +\sum_{i=1}^{N} \lambda_{i}(t)\left[-\left(\rho \sum_{j=1}^{N} a_{i j} B_{j}(t)+\beta_{1}\left(\alpha_{2}+\alpha_{1} \theta_{1}+\alpha_{3} \theta_{2}\right) \sum_{j=1}^{N} B_{j}(t)\right.\right. \\
& \left.\left.+\gamma \sum_{j=1}^{N} a_{i j} R_{j}(t)+\beta_{2}\left(\alpha_{3}\left(1-\theta_{2}\right)\right) \sum_{j=1}^{N} B_{j}(t)\right) T_{i}(t)\right] \\
& +\sum_{i=1}^{N} \mu_{i}(t)\left[\left(\rho \sum_{j=1}^{N} a_{i j} B_{j}(t)+\beta_{1}\left(\alpha_{2}+\alpha_{1} \theta_{1}+\alpha_{3} \theta_{2}\right) \sum_{j=1}^{N} B_{j}(t)\right) T_{i}(t)\right] \\
& +\sum_{i=1}^{N} v_{i}(t)\left[\left(\gamma \sum_{j=1}^{N} a_{i j} R_{j}(t)+\beta_{2}\left(\alpha_{3}\left(1-\theta_{2}\right)\right) \sum_{j=1}^{N} B_{j}(t)\right) T_{i}(t)\right],
\end{aligned}
$$

where $X(t)=(\lambda(t), \mu(t), v(t))=\left(\lambda_{1}, \lambda_{2}, \ldots, \lambda_{N}, \mu_{1}, \mu_{2}\right.$, $\left.\ldots, \mu_{N}, v_{1}, v_{2}, \ldots, v_{N}\right)$ is the adjoint of $H$. We give the necessary condition for the optimal control of the DAS problem as follows.
Theorem 2. Suppose $\theta=\left(\theta_{1}(t), \theta_{2}(t)\right)$ is an optimal control of the DAS problem (16) and $\mathbf{E}$ is the solution to the associated TBR model (17). Then, there exists an adjoint function $X(t)=$ $(\lambda(t), \mu(t), \nu(t))$ such that the following equations hold: 


$$
\left\{\begin{array}{l}
\frac{\mathrm{d} \lambda_{i}(t)}{\mathrm{d} t}=\left(\lambda_{i}-\mu_{i}\right) \sum_{j=1}^{N}\left(\rho a_{i j}+\beta_{1}\left(\alpha_{2}+\alpha_{1} \theta_{1}+\alpha_{3} \theta_{2}\right)\right) B_{j}(t) \\
+\left(\lambda_{i}-v_{i}\right) \sum_{j=1}^{N} \gamma a_{i j} R_{j}+\left(\lambda_{i}-v_{i}\right) \sum_{j=1}^{N} \beta_{2} \alpha_{3}\left(1-\theta_{2}\right) B_{j}, \\
\frac{\mathrm{d} \mu_{i}(t)}{\mathrm{d} t}=-c_{1}+c_{2} \alpha_{1} \theta_{1}+c_{3} \alpha_{3} \theta_{2}+\sum_{j=1}^{N} \rho a_{j i} T_{j}(t)\left(\lambda_{j}-\mu_{j}\right) \\
+\beta_{1}\left(\alpha_{2}+\alpha_{1} \theta_{1}+\alpha_{3} \theta_{2}\right) \sum_{j=1}^{N}\left(\lambda_{j}-\mu_{j}\right) T_{j}(t)+\beta_{2} \alpha_{3}\left(1-\theta_{2}\right) \sum_{j=1}^{N}\left(\lambda_{j}-v_{j}\right) T_{j}(t) \\
\frac{\mathrm{d} v_{i}(t)}{\mathrm{d} t}=\gamma \sum_{j=1}^{N} a_{j i}\left(\lambda_{j}-v_{j}\right) T_{j}(t), \\
0 \leq t \leq T, i=1,2, \ldots, N, \\
\lambda(T)=\mu(T)=v(T)=0 .
\end{array}\right.
$$

Moreover, let

$$
\begin{aligned}
& g_{1}(t)=\beta_{1} \alpha_{1} \sum_{i=1}^{N} T_{i}(t)\left(\mu_{i}-\lambda_{i}\right)-c_{2} \alpha_{1}, \\
& g_{2}(t)=\sum_{i=1}^{N} T_{i}(t)\left(\beta_{1} \alpha_{3}\left(\mu_{i}-\lambda_{i}\right)+\beta_{2} \alpha_{3}\left(\lambda_{i}-v_{i}\right)\right)-c_{3} \alpha_{3} .
\end{aligned}
$$

Then, for $0 \leq t \leq T$, we have

$$
\theta_{i}(t)= \begin{cases}\bar{\theta}_{i}, & g_{i}(t)>0 \\ 0, & g_{i}(t)<0\end{cases}
$$

Proof. According to Pontryagin Minimum Principle [32], there exists $(\lambda, \mu, \nu)$ such that

$$
\begin{cases}\frac{\mathrm{d} \lambda_{i}}{\mathrm{~d} t}=-\frac{\partial H(E(t), \theta(t), X(t))}{\partial T_{i}}, & 0 \leq t \leq T, i=1,2, \ldots, N \\ \frac{\mathrm{d} \mu_{i}}{\mathrm{~d} t}=-\frac{\partial H(E(t), \theta(t), X(t))}{\partial B_{i}}, & 0 \leq t \leq T, i=1,2, \ldots, N \\ \frac{\mathrm{d} \nu_{i}}{\mathrm{~d} t}=-\frac{\partial H(E(t), \theta(t), X(t))}{\partial R_{i}}, & 0 \leq t \leq T, i=1,2, \ldots, N .\end{cases}
$$

The first $3 N$ equations in system (22) follow by direct calculations. Since the terminal cost is unspecified and the final state is free, we have $\lambda(T)=\mu(T)=\nu(T)=0$. According to Pontryagin Maximum Principle, we have
$H(E(t), \theta(t), X(t))=\underset{\bar{\theta} \in \Theta}{\arg \max } H(E(t), \bar{\theta}, X(t)), \quad 0 \leq t \leq T$.

Equations (23) and (24) follow by the following direct calculations:

$$
\begin{aligned}
& \frac{\partial H}{\partial \theta_{1}}=\sum_{j=1}^{N} B_{j}\left(\beta_{1} \alpha_{1} \sum_{i=1}^{N} T_{i}(t)\left(\mu_{i}-\lambda_{i}\right)-c_{2} \alpha_{1}\right), \\
& \frac{\partial H}{\partial \theta_{2}}=\sum_{j=1}^{N} B_{j}\left(\sum_{i=1}^{N} T_{i}(t)\left(\beta_{1} \alpha_{3}\left(\mu_{i}-\lambda_{i}\right)+\beta_{2} \alpha_{3}\left(\lambda_{i}-v_{i}\right)\right)-c_{3} \alpha_{3}\right) .
\end{aligned}
$$

By the optimal control theory, equations (16) and (22) constitute the optimality system for the TBR model. We refer to the control in each solution to the optimality system as a potential optimal control (POC) of the DAS problem. It is seen from equation (24) that the optimality system may have more than one POC.

\section{Examples of the POC}

For the notion of POC we introduced earlier for the DAS problem, now we provide some examples of the POC. First, the optimality system (17) and (22) is solved using the Runge-Kutta and backward Runge-Kutta fourth-order iterative procedure $[34,35]$.

To generate a large number of DAS examples, three realworld networks are selected: Facebook network $G_{\mathrm{FA}}$, e-mail network $G_{\mathrm{EM}}$, and Twitter network $G_{\mathrm{TW}}$. We select the subnet of the network as the total number of nodes of the 
three networks with $N=100$. Let $A_{\mathrm{FA}}, A_{\mathrm{EM}}$, and $A_{\mathrm{TW}}$ denote the adjacency matrix of $G_{\mathrm{FA}}, G_{\mathrm{EM}}$, and $G_{\mathrm{TW}}$, and the network topology is shown in Figure 3. Let the initial value $\mathbf{E}_{0}=\left(T_{0}, B_{0}, R_{0}\right)=(0.7, \ldots, 0.7,0.2, \ldots, 0.2,0.1, \ldots, 0.1)$.

Experiment 1. Stabilities of the three states.

The parameters and their values are shown in Table 3.

As can be seen from Figure 4, in all of three networks, the expected probabilities of the three states are stable at a fixed value. Moreover, when the probability of negative evaluation in the network is larger than that of positive evaluation, nodes tend to refuse to buy the product.

Experiment 2. Optimal control strategies in networks.

Consider the TBR model (17) with $G=G_{\mathrm{FA}}, G=$ $G_{\mathrm{EM}}, G=G_{\mathrm{TW}}, \bar{\theta}_{1}=1, \bar{\theta}_{2}=1, \quad c_{1}=50, c_{2}=5$, and $c_{3}=10$, and the remaining parameters are shown in Table 3. The optimal control $\theta_{p o c}$ is obtained by solving the optimal system, as shown in Figure 5. Figure 6 exhibits $\theta_{\text {poc }} U$ $\theta_{p, q}, p=(0,0.1,0.2, \ldots, 1)$ and $q=(0,0.1,0.2, \ldots, 1)$. It is seen that $J\left(\theta_{\text {poc }}\right)>J\left(\theta_{p, q}\right)$, for all $p, q \in[0,1] . \theta_{\text {poc }}$ is superior to all the uniform controls in the three networks in terms of expected profits. In the above experiment and 100 similar experiments, it is worth emphasizing that $\theta_{\text {poc }}$ outperforms all static controls in terms of expected profits across all the networks, which demonstrates the optimality of $\theta_{\text {poc }}$.

As can be seen from Figure 6, when there is no after-sales service $\left(\theta_{0,0}\right)$ in the community group buying network, the expected profit of sales in the whole network is the lowest due to the influence of negative WOM. When $q=1$, although $p$ is different, the expected profit generated by community group buying is almost the same for the three networks. This phenomenon indicates that reversing negative WOM through after-sales service is more helpful in improving expected profits than operations such as cash back. Moreover, it can be seen from Figures 5 and 6 that the DAS optimal control strategies are concentrated at the initial time, which suggests the importance of timely after-sales service. Delayed or continuous after-sales service has no obvious effect on improving consumers' purchase intention and increasing expected profits.

Experiment 3. Effects of optimal control strategies.

As shown in Figure 7, we compare the expected probabilities of buying and refusing states without control and with the optimal control strategy. It can be inferred that, through DAS optimal control strategies, the probability of buying is greatly increased, while that of refusing buying is greatly decreased. This further proves the importance of after-sales service.

\section{The Influence of Parameters on Expected Profits}

In this section, we discuss how different parameters affect the expected profits. On the one hand, taking node 30 as an example, we discuss the effects of different parameters on purchase intention. On the other hand, given the similar simulation results of the three networks, the e-mail network is taken as an example for illustration, as shown in Figure 8.

Experiment 4. Effects of parameters $\rho, \gamma, \beta_{1}, \beta_{2}$ on purchase intention.

Let $\rho, \gamma, \beta_{1}, \beta_{2}=[0.1,0.3,0.5,0.7,0.9]$ respectively, and the other parameters are shown in Table 3 . The probability of the node's purchase intention increases as $\rho$ and $\beta_{1}$ increase, as shown in Figures 8(a) and 8(c), and decreases as $\gamma$ and $\beta_{2}$ increase, as shown in Figures 8(b) and 8(d). This finding is consistent with the actual representations of the parameters.

Experiment 5. Effects of parameters $\alpha_{1}, \alpha_{2}, \alpha_{3}$ on purchase intention.

Since $\alpha_{1}+\alpha_{2}+\alpha_{3}=1$, let

(1) $\alpha_{1}, \alpha_{2}=[0.1,0.2,0.3,0.4,0.5]$ and $\alpha_{3}=1-\alpha_{1}-\alpha_{2}$

(2) $\alpha_{1}, \alpha_{3}=[0.1,0.2,0.3,0.4,0.5]$ and $\alpha_{2}=1-\alpha_{1}-\alpha_{3}$

(3) $\alpha_{2}, \alpha_{3}=[0.1,0.2,0.3,0.4,0.5]$ and $\alpha_{1}=1-\alpha_{2}-\alpha_{3}$

When $T=25$, take the probability of buying for node 30 as an example. As shown in Figure 9(a), when $\alpha_{2}$ is very small, the probability changes little with the increase of $\alpha_{1}$. Specifically, despite the increased probability of silent purchase, the probability of final node purchase does not increase significantly. This is because the probability of positive WOM is fixed at a small value. When $\alpha_{2}$ is large, the probability increases significantly as $\alpha_{1}$ increases. When the probability of positive WOM is fixed at a large value, the probability of negative WOM decreases as the probability of silent purchase increases, so the probability of final node purchase increases significantly. The situation in Figure 9(b) is similar to that in Figure 9(a). It can be clearly seen from Figure 9(c) that the purchase probability increases with the increase of $\alpha_{2}$ and decreases with the increase of $\alpha_{3}$.

Under optimal dynamic after-sales service control, the expected profit exhibits different levels of sensitivity to different parameters. Therefore, we will further discuss how the parameters affect the expected profit of the entire community group buying network later in this section.

Experiment 6. Effects of parameters $\rho, \gamma, \beta_{1}, \beta_{2}$ on the expected profit.

Let $\rho, \gamma, \beta_{1}, \beta_{2}=[0.1,0.3,0.5,0.7,0.9]$, respectively, and the other parameters are shown in Table 3 . As can be seen from Figure 10, the expected profit of the whole network increases with the increase of $\rho, \beta_{1}$ and decreases with the increase of $\gamma, \beta_{2}$ after-sales service is available. Based on Figure 8 , it can be concluded that when consumers' purchase intention increases, the expected profit also increases in the network.

Experiment 7. Effects of parameters $\alpha_{1}, \alpha_{2}, \alpha_{3}$ on expected profits.

Since $\alpha_{1}+\alpha_{2}+\alpha_{3}=1$, let

(1) $\alpha_{1}, \alpha_{2}=[0.1,0.2,0.3,0.4,0.5]$ and $\alpha_{3}=1-\alpha_{1}-\alpha_{2}$

(2) $\alpha_{1}, \alpha_{3}=[0.1,0.2,0.3,0.4,0.5]$ and $\alpha_{2}=1-\alpha_{1}-\alpha_{3}$

(3) $\alpha_{2}, \alpha_{3}=[0.1,0.2,0.3,0.4,0.5]$ and $\alpha_{1}=1-\alpha_{2}-\alpha_{3}$ 


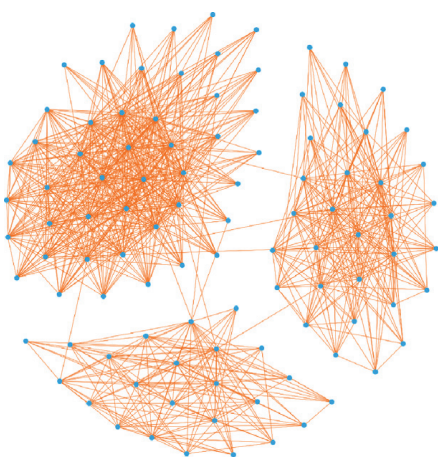

(a)

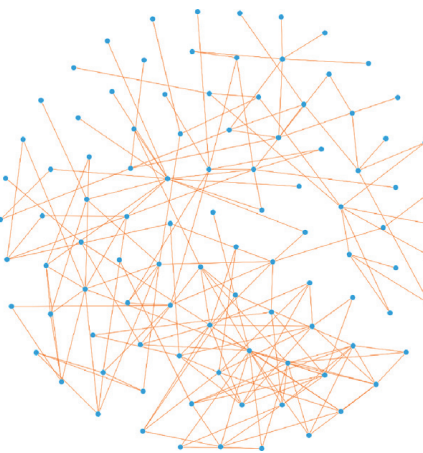

(b)

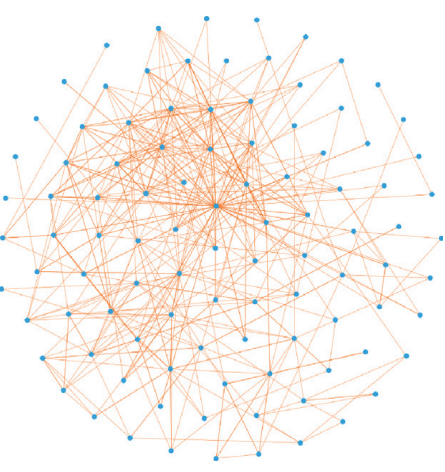

(c)

Figure 3: (a) Facebook network, (b) e-mail network, and (c) Twitter network.

TABle 3: Parameters based on the network

\begin{tabular}{lcccccccc}
\hline Parameters & $\rho$ & $\gamma$ & $\alpha_{1}$ & $\alpha_{2}$ & $\alpha_{3}$ & $\beta_{1}$ & $\beta_{2}$ & $T$ \\
\hline Value & 0.1 & 0.2 & 0.4 & 0.2 & 0.4 & 0.1 & 0.1 & 25 \\
\hline
\end{tabular}

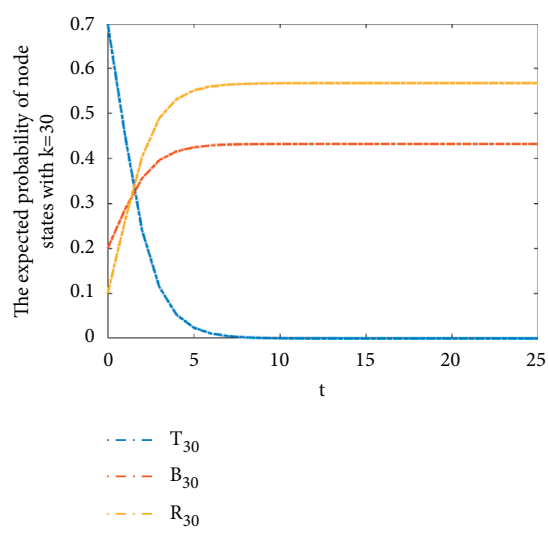

(a)

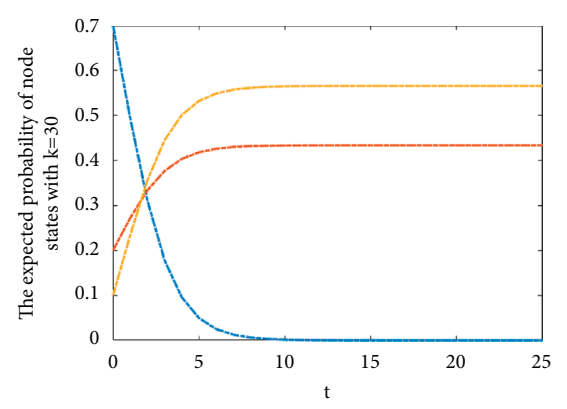

$\begin{array}{ll}\cdot-\cdot-\mathrm{T}_{30} \\ -\cdot- & \mathrm{B}_{30} \\ -\cdot- & \mathrm{R}_{30}\end{array}$

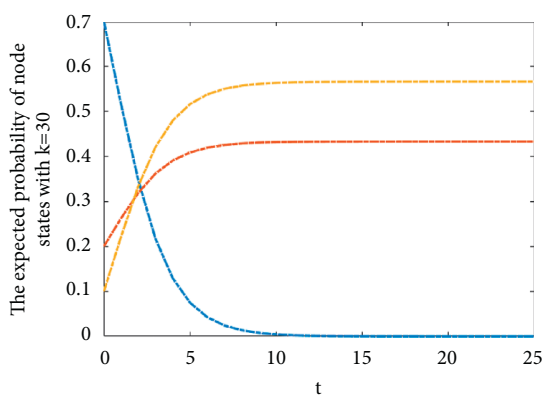

-..- $\mathrm{T}_{30}$

$\cdot-\cdot \mathrm{B}_{30}$

$\cdots \mathrm{R}_{30}$

(c)

FIgURE 4: (a) Facebook network, (b) e-mail network, and (c) Twitter network.

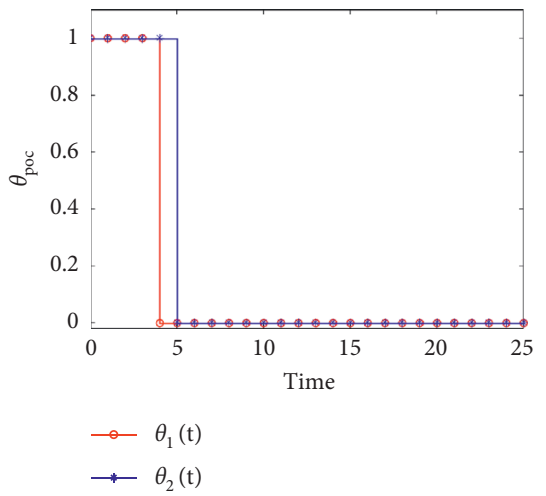

(a)

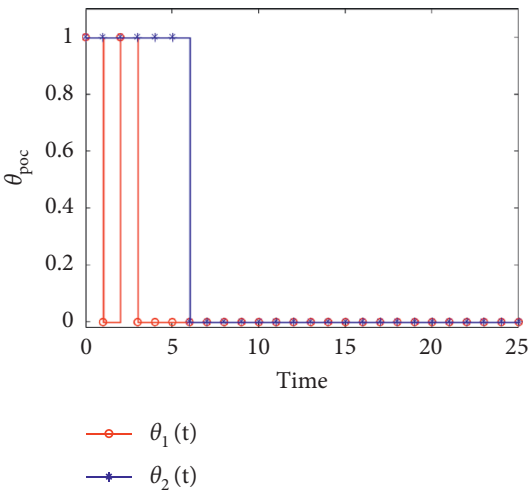

(b)

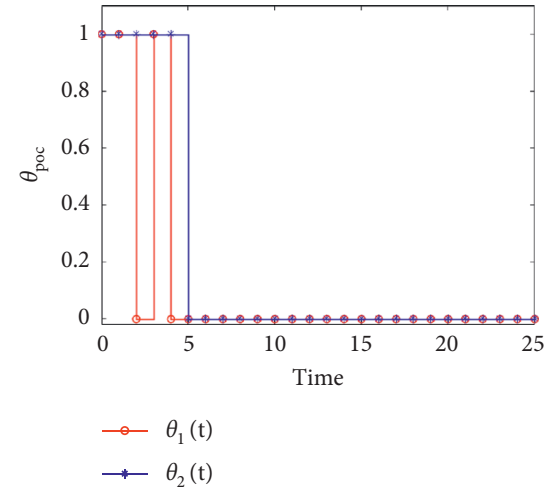

(c)

FIgURE 5: Optimal control strategies $\theta_{\text {poc }}$ in networks: (a) Facebook network, (b) e-mail network, and (c) Twitter network. 


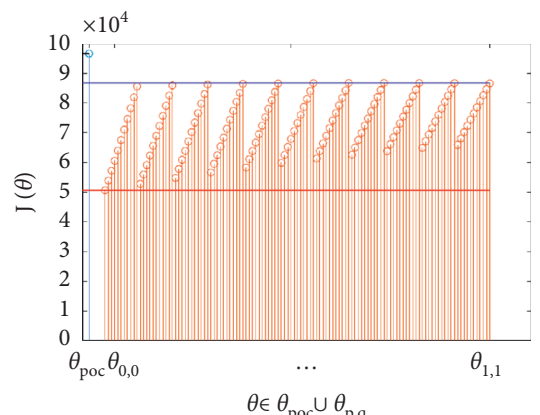

(a)

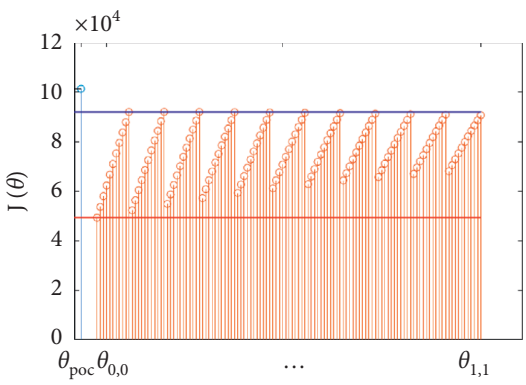

$\theta \in \theta_{\mathrm{poc}} \cup \theta_{\mathrm{p}, \mathrm{q}}$

(b)

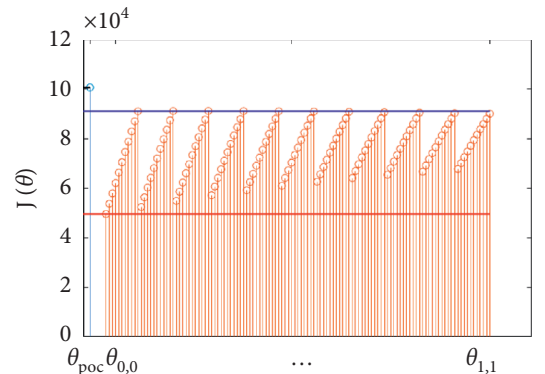

$\theta \in \theta_{\mathrm{poc}} \cup \theta_{\mathrm{p}, \mathrm{q}}$

(c)

FIGURE 6: Comparison between optimal control strategy $\theta_{\text {poc }}$ and uniform control strategy $\theta_{p, q}$ in terms of expected profit $J(\theta)$ : (a) Facebook network, (b) e-mail network, and (c) Twitter network.

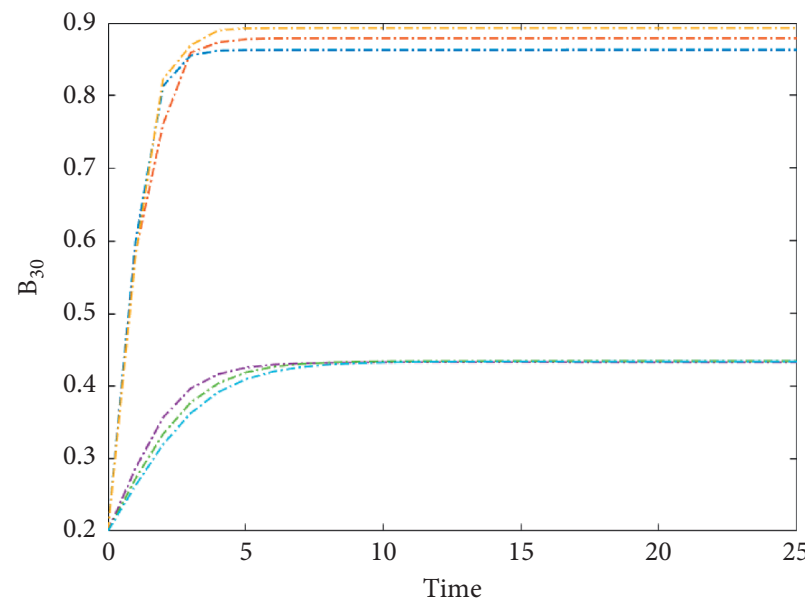

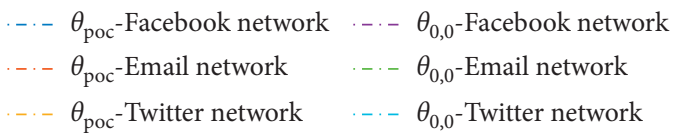

(a)

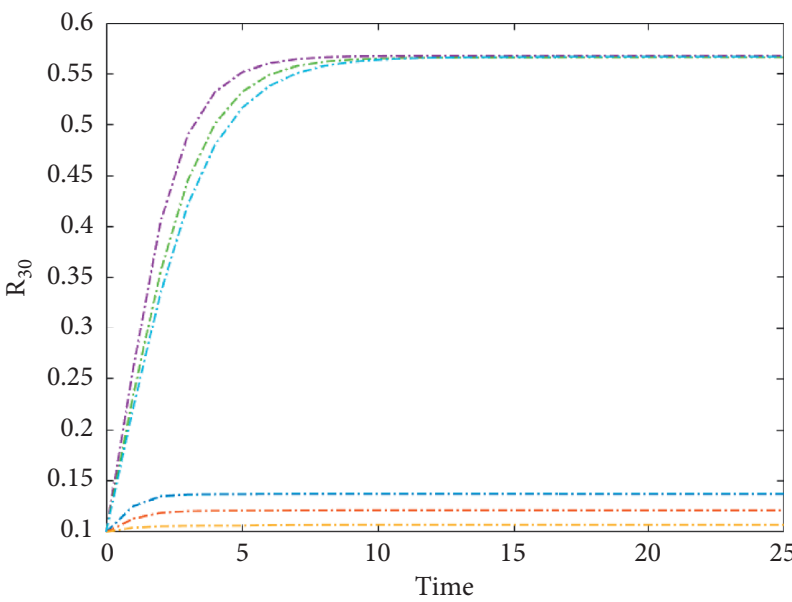

-.- $\theta_{\text {poc }}$-Facebook network -..- $\theta_{0,0}$-Facebook network

...- $\theta_{\text {poc }}$-Email network _..- $\theta_{0,0}$-Email network

$\theta_{\text {poc }}$-Twitter network _... $\theta_{0,0}$-Twitter network

(b)

Figure 7: (a) Expected probability of buying for node 30 under $\theta_{\text {poc }}$ and without control $\theta_{0,0}$ and (b) expected probability of refusing for node 30 under $\theta_{\text {poc }}$ and without control $\theta_{0,0}$.

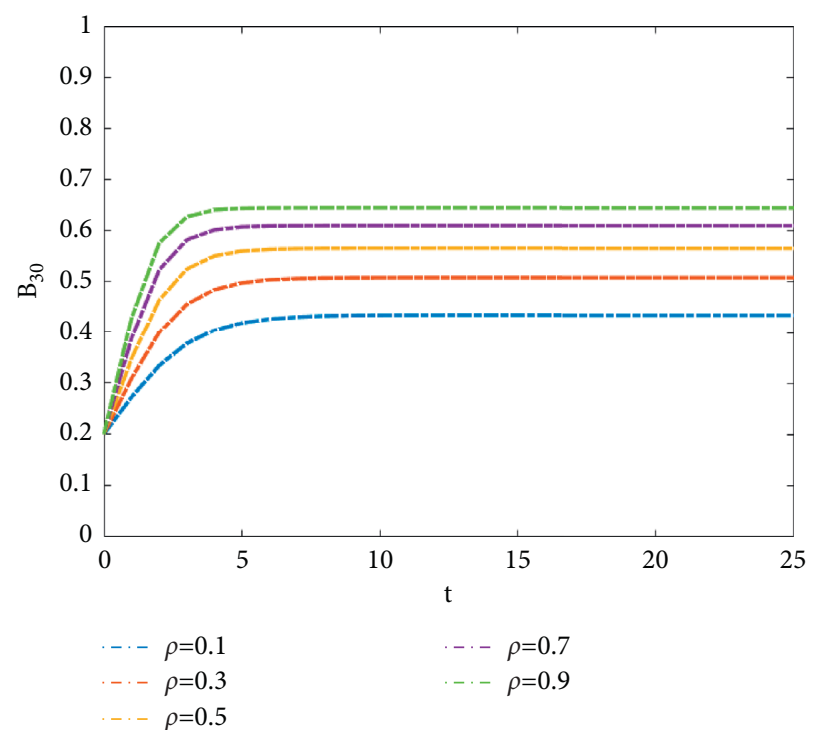

(a)

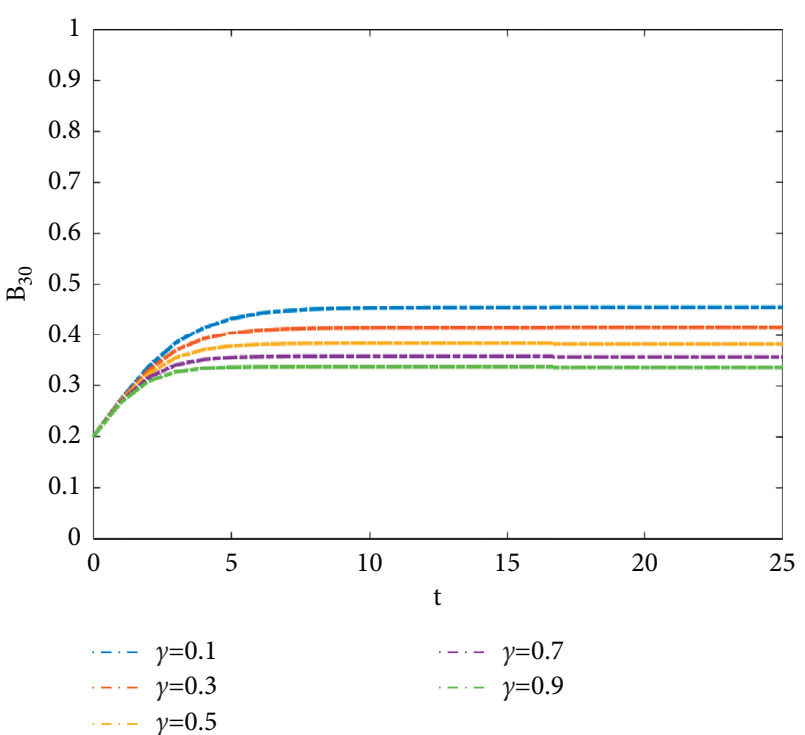

(b)

Figure 8: Continued. 


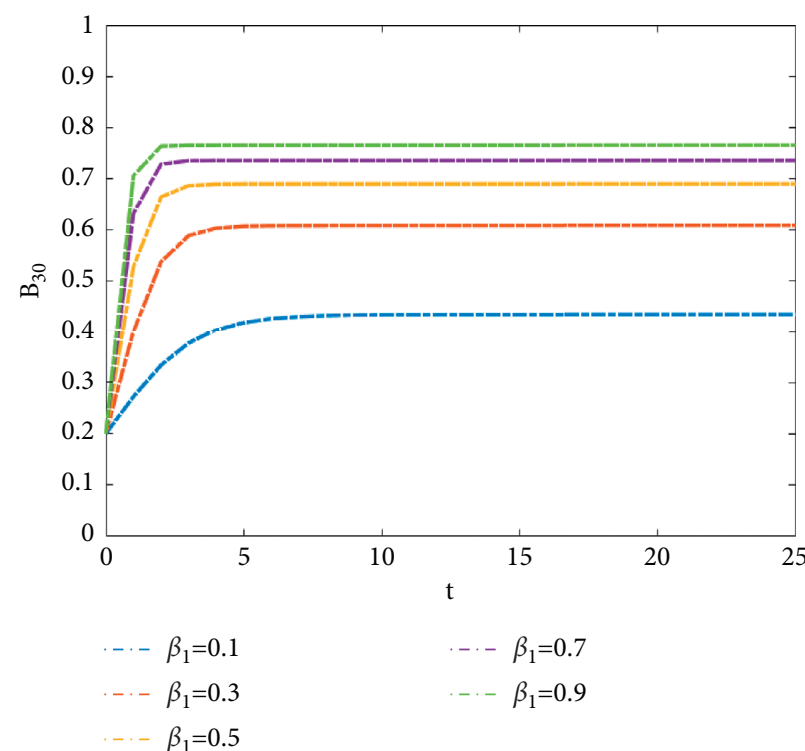

(c)

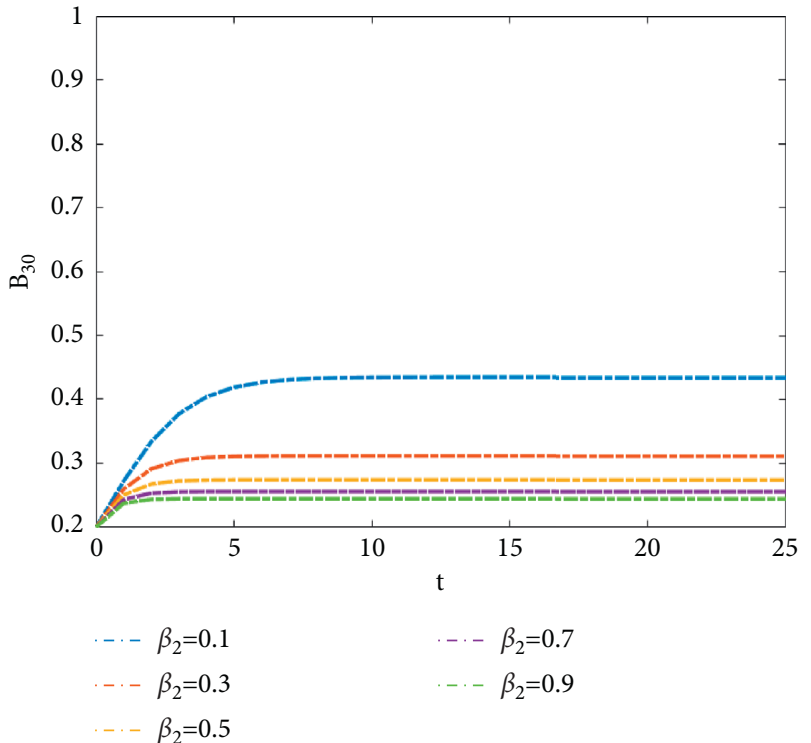

(d)

Figure 8: Parameters on the expected probability of buying for node 30: (a) $\rho$, (b) $\gamma$, (c) $\beta_{1}$, and (d) $\beta_{2}$.

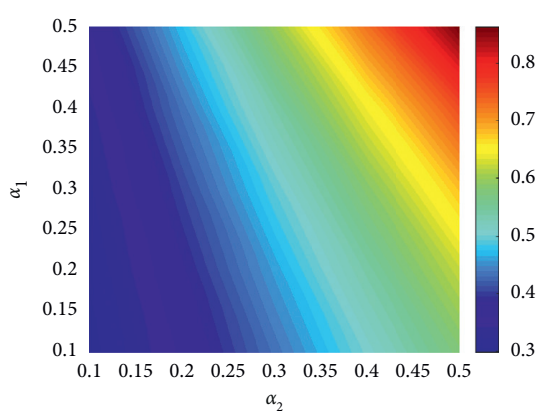

(a)

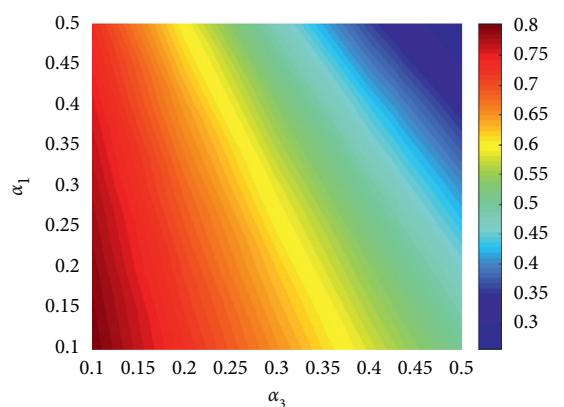

(b)

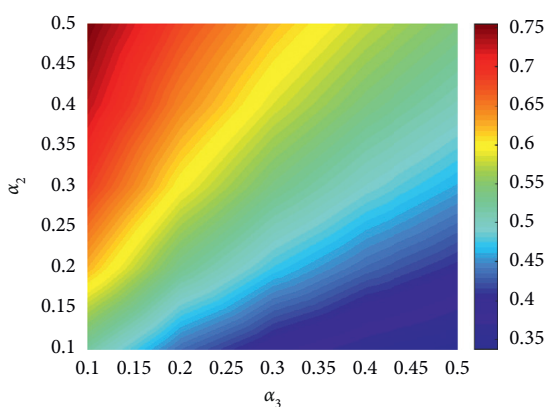

(c)

Figure 9: Parameters on the expected probability of buying for node 30: (a) $\alpha_{1}, \alpha_{2}$, (b) $\alpha_{1}, \alpha_{3}$, and (c) $\alpha_{2}, \alpha_{3}$.

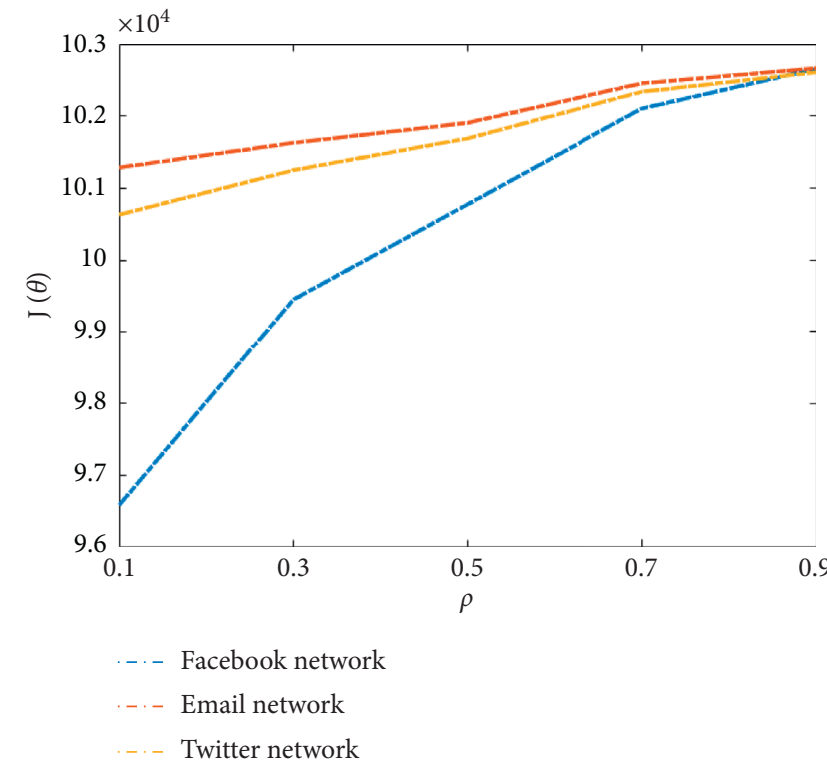

(a)

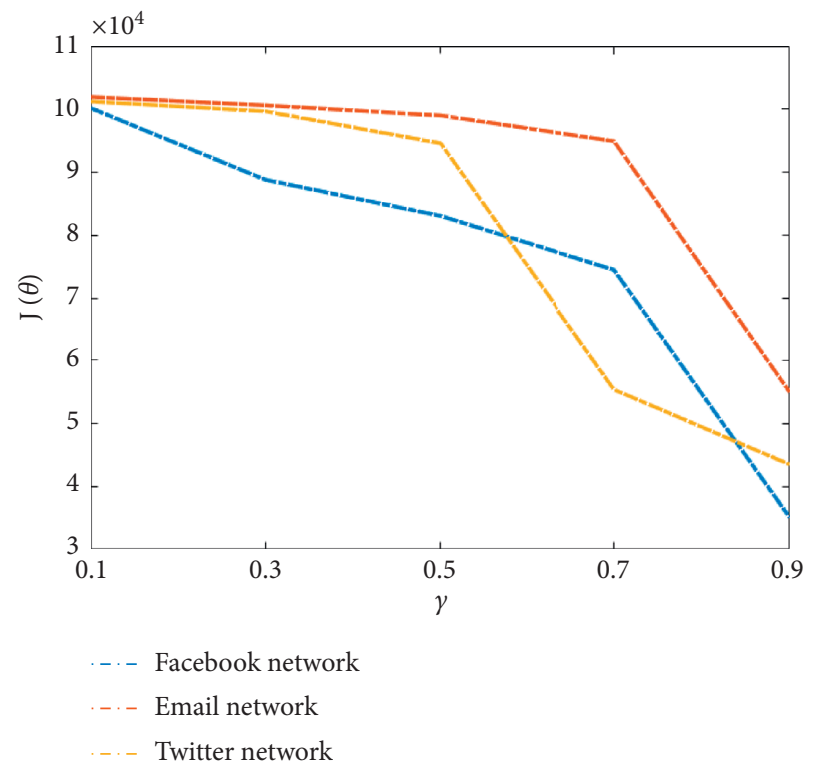

(b)

FIgURe 10: Continued. 


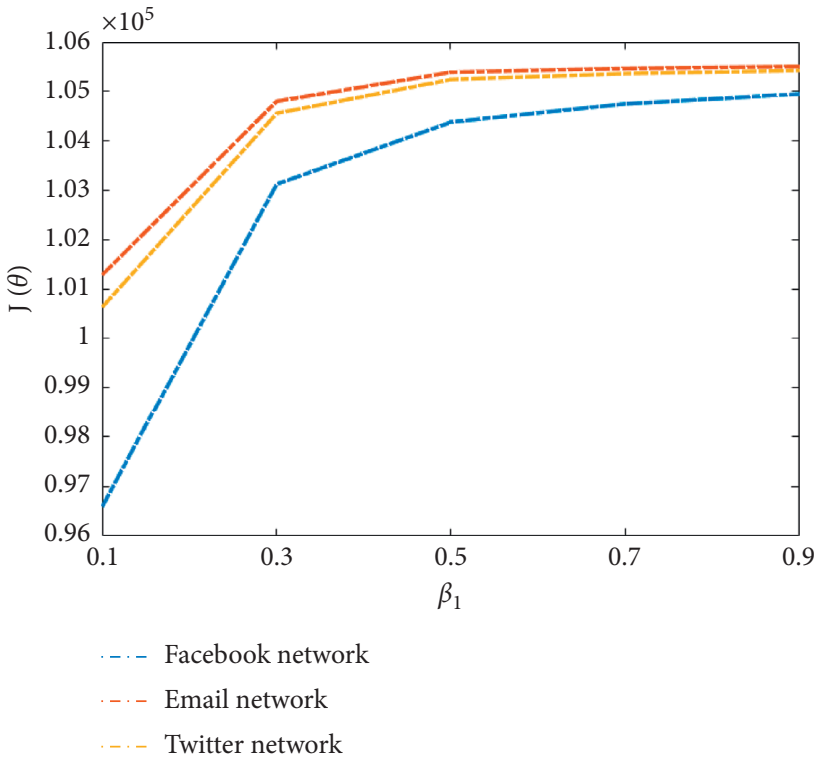

(c)

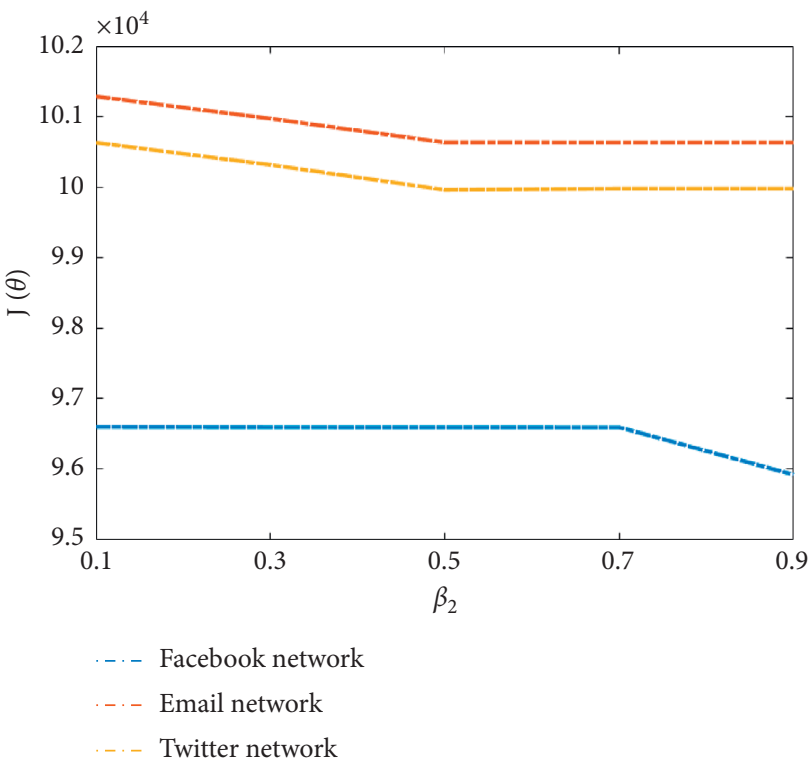

(d)

FIGURE 10: Parameters on the expected probability of buying for node 30 : (a) $\rho$, (b) $\gamma$, (c) $\beta_{1}$, and (d) $\beta_{2}$.

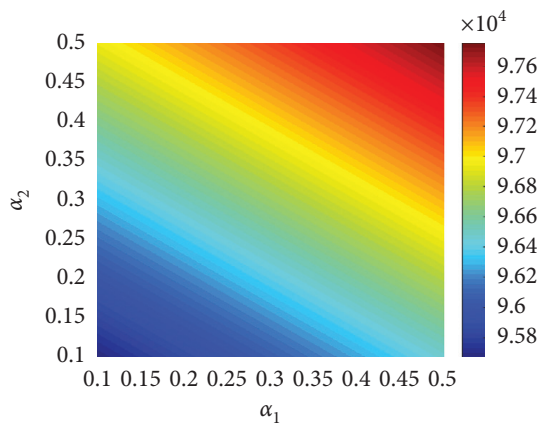

(a)

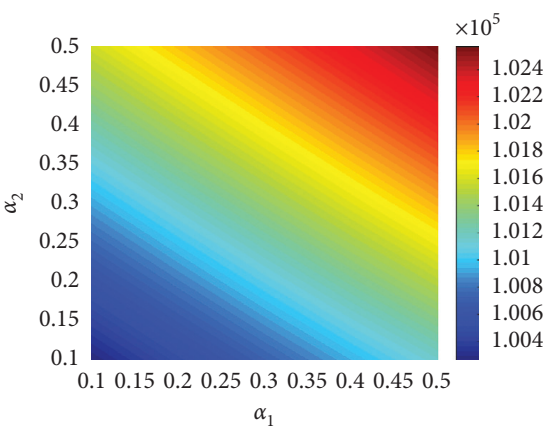

(d)

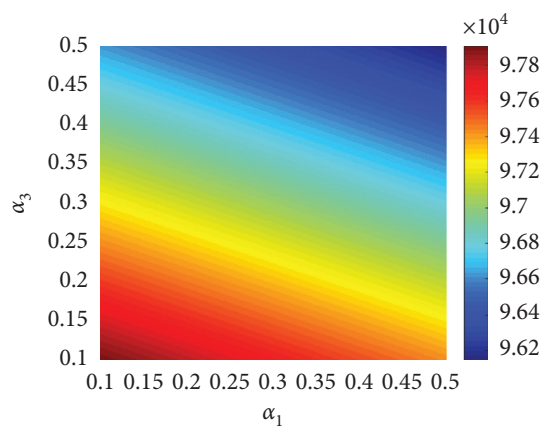

(b)

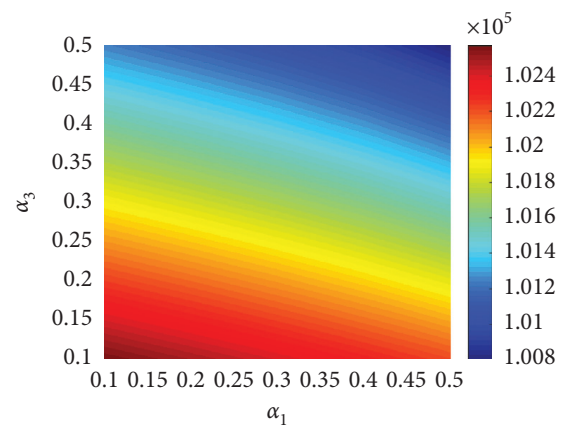

(e)

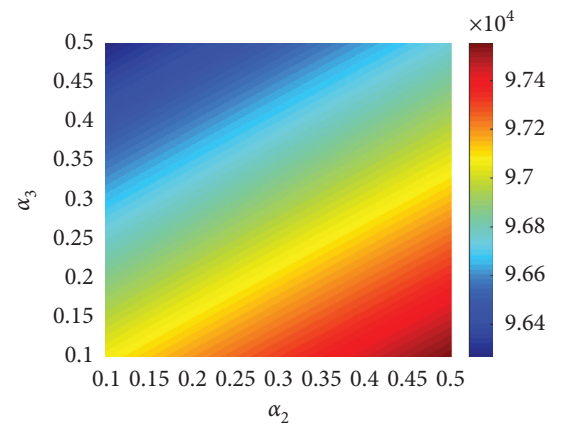

(c)

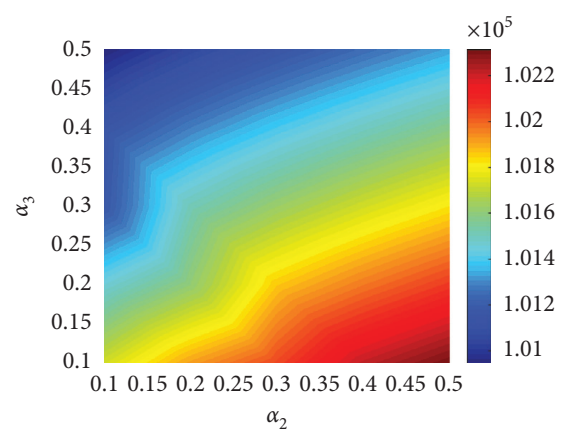

(f)

FIgURE 11: Continued. 


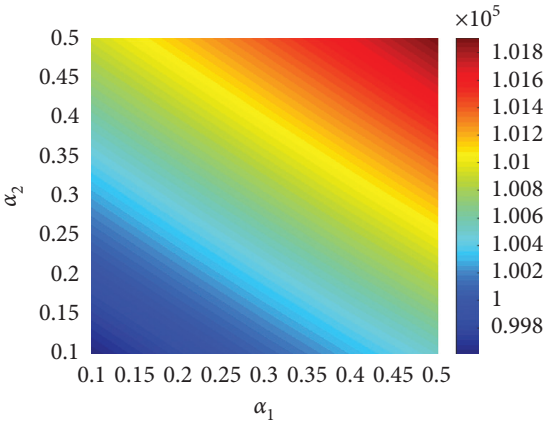

(g)

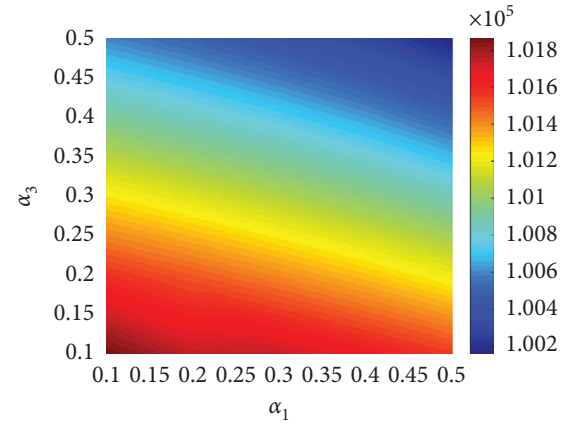

(h)

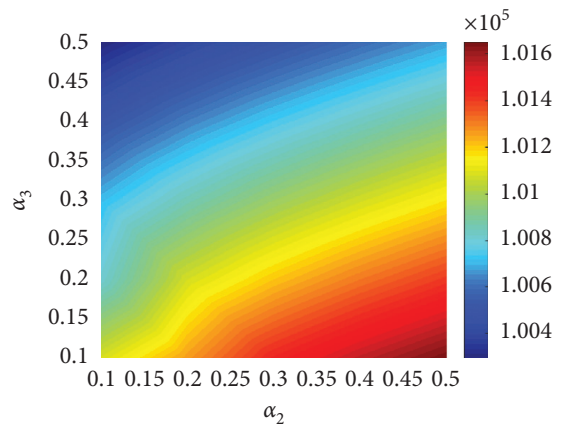

(i)

Figure 11: Parameters $\alpha_{1}, \alpha_{2}, \alpha_{3}$ on the expected profit. (a-c) $\alpha_{1}, \alpha_{2}, \alpha_{3}$ on Facebook network; (d-f) $\alpha_{1}, \alpha_{2}, \alpha_{3}$ on e-mail network; (g-i) $\alpha_{1}, \alpha_{2}, \alpha_{3}$ on Twitter network.

TABLE 4: Summary of experimental conclusion.

\begin{tabular}{|c|c|c|}
\hline Number & Conclusion & $\begin{array}{l}\text { Supporting } \\
\text { experiments }\end{array}$ \\
\hline$C_{1}$ & The three states in the network will stabilize to & Experiment 1 \\
\hline$C_{2}$ & $\begin{array}{l}\text { timal control strategy outperforms any uniform control strategy and can greatly improve the } \\
\text { expected profit }\end{array}$ & Experiments \\
\hline$C_{3}$ & Reversing negative reviews is more helpful in improving expected profits than improve silent evaluation & nt 2 \\
\hline $\mathrm{C}_{4}$ & & Experin \\
\hline$C_{5}$ & The probability $\gamma$ of $T_{i}$ being converted to $R_{i}$ is negatively correlated with expected profits & Experimen \\
\hline$C_{6}$ & The probability of positive evaluation $\alpha_{2}$ is positively correlated with expected profits & Experiment \\
\hline$C_{7}$ & The probability of negative evaluation $\alpha_{3}$ is positi & Experin \\
\hline$C_{8}$ & Probability $\beta_{1}$ influe & Experi \\
\hline$C_{9}$ & Probability $\beta_{2}$ influenced by negative WOM is negatively correlated with expected profits & Experiments 4 and 6 \\
\hline$C_{10}$ & $\begin{array}{l}\text { When the probability } \alpha_{2}\left(\alpha_{3}\right) \text { of positive (negative) WOM is fixed and small, the probability of silent } \\
\text { evaluation } \alpha_{1} \text { has a weak positive (negative) correlation with purchase intention }\end{array}$ & Experiment 5 \\
\hline$C_{11}$ & $\begin{array}{c}\text { When the probability } \alpha_{2}\left(\alpha_{3}\right) \text { of positive (negative) WOM is fixed and large, the probability of silent } \\
\text { evaluation } \alpha_{1} \text { has a strong positive (negative) correlation with purchase intention }\end{array}$ & Exp \\
\hline
\end{tabular}

As can be seen from Figure 11, the expected profit is not closely related to the proportion of silent customers, but is directly proportional to positive WOM and inversely proportional to negative WOM. This conclusion is consistent with the analysis in Figure 9.

The experimental conclusions are summarized in Table 4 .

\section{Conclusion}

This paper aims at maximizing the expected profits of product sales in social network shopping by dynamically controlling the proportion of after-sales service investment based on word-of-mouth marketing. First, the problem is modeled as an optimal control problem. Second, a potential optimal control strategy is proposed and the optimal control problem is identified by solving the optimal system. Finally, some comparative experiments demonstrate that the proposed dynamic control strategy is superior to any uniform control strategy, and the expected profits show different levels of sensitivity to different after-sales service parameters. Therefore, the proposed dynamic after-sales investment strategy can serve as an effective approach to maximizing the expected profits of product sales in social network shopping. In this regard, there are a number of research topics worthy of further study. The problem of balancing profits and after-sales investment can be modeled as a game theory problem to ensure maximum interests for individuals.

\section{Data Availability}

The data used to support the findings of this study are included within the article.

\section{Conflicts of Interest}

The authors declare no potential conflicts of interest.

\section{Acknowledgments}

This work was supported by the National Natural Science Foundation of China, under Grant nos. 61807028, 61772449, 61572420, and 61802332, and Natural Science Foundation of Hebei Province China, under Grant no. F2019203120. 


\section{References}

[1] "Accenture consulting," Report of Chinese Consumer Insight by Accenture, Dublin, Ireland, 2014.

[2] Dichter, "How word-of-mouth advertising works," Harvard Business Review, vol. 16, 1966.

[3] J. Chevalier, The Effect of Word of Mouth on Sales: Online Book Reviews, Working Papers - Yale School of Management's Economics Research Network, Connecticut, CT, USA, 2003.

[4] L. Leskovec, "Adamic, he dynamics of viral marketing," ACM Transactions on the Web, vol. 1, 2005.

[5] S. Hill, F. Provost, and C. Volinsky, Viral Marketing: Identifying Likely Adopters via Consumer Networks, Social Science Electronic Publishing, New York, NY, USA, 2005.

[6] S. Hill, F. Provost, and C. Volinsky, "Network-based marketing: identifying likely adopters via consumer networks," Statistical Science, vol. 21, pp. 256-276, 2006.

[7] A. Vilpponen, S. Winter, and S. Sundqvist, "Network-based marketing: identifying likely adopters via consumer networks," Statistical Science, vol. 21, pp. 256-276, 2006.

[8] J. Brown, A. J. Broderick, and N. Lee, "Word of mouth communication within online communities: conceptualizing the online social network," Journal of Interactive Marketing, vol. 21, no. 3, pp. 2-20, 2007.

[9] D. D. Gunawan and K.-H. Huarng, "Viral effects of social network and media on consumers' purchase intention," Journal of Business Research, vol. 68, no. 11, pp. 2237-2241, 2015.

[10] D. Godes and D. Mayzlin, "Firm-created word-of-mouth communication: evidence from a field test," Marketing Science, vol. 28, no. 4, pp. 721-739, 2009.

[11] J. Zhang, Y. Liu, and Y. Chen, Social Learning in Networks of Friends versus Strangers, Social Science Electronic Publishing, New York, NY, USA, 2015.

[12] D. Dubois, A. Bonezzi, and M. De Angelis, "Sharing with friends versus strangers: how interpersonal closeness influences word-of-mouth valence," Journal of Marketing Research, vol. 53, no. 5, pp. 712-727, 2016.

[13] A. Timoshenko and J. R. Hauser, "Identifying customer needs from user-generated content," Marketing Science, vol. 38, 2019.

[14] Z. A. Min, B. Bf, Z. C. Ning, and E. Wf, "Mining product innovation ideas from online reviews," Information Processing \& Management, vol. 58, 2021.

[15] R. Y. Du, O. Netzer, D. A. Schweidel, and D. Mitra, "Capturing marketing information to fuel growth," Journal of Marketing, vol. 85, no. 1, pp. 163-183, 2021.

[16] T. Zhang, P. Li, L.-X. Yang, X. Yang, Y. Y. Tang, and Y. Wu, "A discount strategy in word-of-mouth marketing," Communications in Nonlinear Science and Numerical Simulation, vol. 74, pp. 167-179, 2019.

[17] J. Chen, L. X. Yang, D. W. Huang, X. Yang, and Y. Y. Tang, "Dynamic discount pricing in competitive marketing," IEEE Access, vol. 7, pp. 14534-145347, 2019.

[18] H. Peng, K. Huang, L.-X. Yang, X. Yang, and Y. Y. Tang, "Dynamic maintenance strategy for word-of-mouth marketing," IEEE Access, vol. 8, pp. 126496-126503, 2020.

[19] M. Herrera, G. Armelini, and E. Salvaj, "Understanding social contagion in adoption processes using dynamic social networks," PLoS One, vol. 10, no. 10, Article ID e0140891, 2015.

[20] M. Wu, L. Wang, L. Ming, and H. Long, "An approach based on the SIR epidemic model and a genetic algorithm for optimizing product feature combinations in feature fatigue analysis," Journal of Intelligent Manufacturing, vol. 26, pp. 1-11, 2015.

[21] M. Pazoki and H. Samarghandi, "Word-Of-Mouth and estimating demand based on network structure and epidemic models," European Journal of Operational Research, vol. 291, 2021.

[22] S. Soobin and J. SooCheong, "A negative or positive signal? The impact of food recalls on negative word-of-mouth $(\mathrm{N}$ WOM)," Journal of Hospitality and Tourism Management, vol. 47, pp. 150-158, 2021.

[23] F. Septianto, G. Northey, T. M. Chiew, and L. Ngo, "Hubristic pride \& prejudice: the effects of hubristic pride on negative word-of-mouth," International Journal of Research in Marketing, vol. 37, 2020.

[24] H. H. Chang, Y.-C. Tsai, K. H. Wong, J. W. Wang, and F. J. Cho, "The effects of response strategies and severity of failure on consumer attribution with regard to negative wordof-mouth," Decision Support Systems, vol. 71, pp. 48-61, 2015.

[25] P. Matthew and A. Laurence, "I should have known better!: when firm-caused failure leads to self-image concerns and reduces negative word-of-mouth," Journal of Business Research, vol. 116, 2020.

[26] C. Riza and S. Hyunju, "The effects of harm directions and service recovery strategies on customer forgiveness and negative word-of-mouth intentions," Journal of Retailing and Consumer Services, vol. 27, pp. 103-112, 2015.

[27] M. S. Balaji, K. W. Khong, and A. Chong, "Determinants of negative word-of-mouth communication using social networking," Information \& Management, vol. 53, 2016.

[28] T. Verhagen, A. Nauta, and F. Feldberg, "Negative online word-of-mouth: behavioral indicator or emotional release?" Serie Research Memoranda, vol. 29, pp. 1430-1440, 2012.

[29] M. D. Dalman, S. Chatterjee, and J. Min, "Negative word of mouth for a failed innovation from higher/lower equity brands: moderating roles of opinion leadership and consumer testimonials," Journal of Business Research, vol. 115, pp. 1-13, 2020.

[30] W. Weitzl, C. Hutzinger, and S. Einwiller, "An empirical study on how webcare mitigates complainants' failure attributions and negative word-of-mouth," Computers in Human Behavior, vol. 89, pp. 316-327, 2018.

[31] E. M. Stein and R. Shakarchi, Real Analysis: Measure Theory, Integration, \& Hilbert Spaces, Princeton University Press, New Jersey, NJ, USA, 2005.

[32] D. Liberzon, Calculus of Variations and Optimal Control Theory: A Concise Introduction, Princeton University Press, New Jersey, NJ, USA, 2012.

[33] R. C. Robinson, Calculus of Variations and Optimal Control Theory: A Concise an Introduction to Dynamical Systems: Continuous and Discrete, American Mathematical Society, Providence, RI, USA, 2004.

[34] S. Lenhart and J. T. Workman, Optimal Control Applied to Biological Models, Champion and Hall/CRC, London, UK, 2007.

[35] J. T. K. Soovoojeet, "A mathematical study of a prey-predator model in relevance to pest control," Nonlinear Dynamics, vol. 74, no. 3, pp. 667-683, 2013. 